\title{
Postoperative adjuvant radiotherapy is associated with improved survival in hepatocellular carcinoma with microvascular invasion
}

\author{
Liming Wang ${ }^{1, *}$, Weihu Wang ${ }^{2,}{ }^{*}$, Xuesong Yao ${ }^{3}$, Weiqi Rong ${ }^{1}$, Fan Wu${ }^{1}$, Bo Chen ${ }^{4}$, \\ Mei Liu ${ }^{5}$, Shengtao Lin ${ }^{1}$, Yunhe Liu ${ }^{1}$ and Jianxiong $\mathbf{W} \mathbf{u}^{1}$ \\ ${ }^{1}$ Department of Hepatobiliary Surgery, National Cancer Center/Cancer Hospital, Chinese Academy of Medical Sciences and \\ Peking Union Medical College, Beijing, China \\ ${ }^{2}$ Department of Radiation Oncology, Key Laboratory of Carcinogenesis and Translational Research (Ministry of Education/ \\ Beijing), Peking University Cancer Hospital and Institute, Beijing, China \\ ${ }^{3}$ Department of Interventional Therapy, National Cancer Center/Cancer Hospital, Chinese Academy of Medical Sciences and \\ Peking Union Medical College, Beijing, China \\ ${ }^{4}$ Department of Radiation Oncology, National Cancer Center/Cancer Hospital, Chinese Academy of Medical Sciences and \\ Peking Union Medical College, Beijing, China \\ ${ }^{5}$ Laboratory of Cell and Molecular Biology and State Key Laboratory of Molecular Oncology, National Cancer Center/Cancer \\ Hospital, Chinese Academy of Medical Sciences and Peking Union Medical College, Beijing, China \\ *These authors contributed equally to this work
}

Correspondence to: Jianxiong Wu, email: dr.wujx@hotmail.com

Keywords: hepatocellular carcinoma, microvascular invasion, TACE, radiotherapy, relapse-free survival

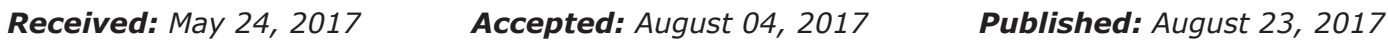

Copyright: Wang et al. This is an open-access article distributed under the terms of the Creative Commons Attribution License 3.0 (CC BY 3.0), which permits unrestricted use, distribution, and reproduction in any medium, provided the original author and source are credited.

\section{ABSTRACT}

Purpose: Limited studies have compared the efficacy of postoperative adjuvant therapies in HCC patients with microvascular invasion (MVI). In this study we assess the efficacy of postoperative adjuvant conservative therapy (CT), trans-catheter arterial chemoembolization (TACE) and radiotherapy (RT) in HCC patients with MVI.

Results: Kaplan-Meier survival analysis revealed that patients in the RT group have significantly improved RFS (RT vs TACE: $p=0.011$; RT vS CT: $p<0.001$ ) and OS (RT vs. TACE: $p=0.034$; RT vs CT: $P<0.001$ ) compared to TACE and CT groups. Further, subgroup analysis based on the degree of MVI and surgical margin width showed that patients with narrow surgical margin have significantly longer RFS and OS after adjuvant RT than the TACE and CT, independent of degree of MVI. Multivariate analysis indicated that MVI classification is the independent prognostic factor associated with RFS and OS.

Materials and Methods: Between July 2008 and December 2015, 136 HCC patients with MVI were divided into three groups according to their adjuvant therapies. Survival outcomes namely relapse-free survival (RFS) and overall survival (OS) of the three groups were analyzed.

Conclusions: Adjuvant radiotherapy following hepatectomy could result in better survival outcomes for HCC patients with MVI than TACE or CT.

\section{INTRODUCTION}

Hepatocellular carcinoma (HCC), the primary malignancy of the liver, is the sixth most common cancer and the second leading cause of cancer-related mortality in the world [1]. It is of major concern in less developed countries where it accounts for $83 \%$ of the estimated 782,000 new cancer cases $[1,2]$. Eastern Asia and sub-
Saharan Africa reported the highest age adjusted incidence rate of over 20 per 100,000 individuals [3]. In China, primary liver cancer is the second most common cancer and accounts for $55 \%$ of all HCC cases, [2] and nearly 383,203 persons die of liver cancer every year and this accounts for $51 \%$ of liver cancer deaths worldwide [4]. Cirrhosis caused by hepatitis $\mathrm{B}$ and hepatitis $\mathrm{C}$ virus infections is the main risk factor for HCC [2-4]. Surgical 
resection and liver transplantation are the standard of care curative treatment modalities for HCC in cirrhotic patients with good functional reserves [5]. However, postoperative intrahepatic recurrence of HCC remains a significant clinical problem with recurrence rate as high as $70 \%-100 \%$ at 5 -years after resection and $15-30 \%$ after liver transplantation $[6,7]$. The various risk factors for recurrence of HCC include tumor size, tumor number, vascular invasion (both macroscopic and microscopic), presence of stellate nodules, histopathological grade, underlying cirrhosis and the type of surgery (i.e. narrow vs. wide surgical margins, anatomic vs. non-anatomic resection, minor vs. major resections) [6].

Microvascular invasion (MVI), is the most important risk factor that is significantly associated with early postoperative recurrence in the liver remnant and is confirmed as an independent predictor of both overall and disease-free survival after liver resection [7-9]. Even for patients with small HCCs, MVI increase the rate of tumor recurrence and dramatically shortens long-term survival $[10,11]$. Unfortunately, MVI can be detected only by postoperative histological examination, which makes the postoperative treatment an essential requirement.

The effectiveness of adjuvant radiotherapy in reducing the recurrence and improving the overall survival is well documented in clinical studies $[12,13]$. Earlier study has shown that postoperative intensity-modulated radiation therapy (IMRT) improved 3-year overall and disease-free survival in HCC patients receiving narrow margin hepatectomy close to major vessels [12]. In addition, the post-hoc subgroup comparison of randomized trial also demonstrated that adjuvant radiotherapy improved recurrence-free survival in patients with small HCC lesions [13]. Although, various adjuvant therapies such as TACE, radiation therapy, interferon, polyprenoic acid, adoptive immunotherapy and iodine-131-labeled lipiodol were used to decrease the recurrence and prolong the survival; it is important to note that studies comparing these adjuvant therapies taking the MVI into consideration are scarce. Consequently, the optimal postoperative treatment algorithms for preventing recurrence of $\mathrm{HCC}$ in patients with MVI have not been thoroughly evaluated. Therefore, in this retrospective, single-center study, we analyze the survival outcomes of HCC patients with MVI who received conservative treatment, radiotherapy or TACE as their primary adjuvant treatment after hepatectomy and analyzed the variables influencing the outcomes in these patients.

\section{RESULTS}

\section{Demographic and clinicopathological characteristics}

A total of 136 patients (118 male, 18 female) with a mean age of $53.51 \pm 11.36$ years (range 27-79) were included in the study (CT group, $N=50$; TACE group, $N=42$; RT group, $N=44$ ). The baseline demographic and the clinicopathological characteristics of the three groups of patients with MVI are summarized in Table 1. Most of the characteristics of the three groups were comparable. Patients in the CT group were significantly older ( $p=0.014)$ and those in RT group had significantly higher rates of narrow surgical margin $(p=0.010)$ than the other two groups.

\section{Relapse-free survival and overall survival rates of the CT, TACE and RT groups}

The 1-, 2-, and 3-year RFS rates were 37.4, 14.8 and $11.1 \%$ for patients in the CT group, 36.2, 30.7 and $26.8 \%$ for patients in the TACE group, 66.7, 52.8, and 45.5\% for patients in the RT group, respectively. The 1-, 2-, and 3 -year OS rates were $69.0,50.4$, and $28.1 \%$ for patients in the CT group, 86.7, 53.0, and $43.7 \%$ for patients in the TACE group and $90.2,80.6$, and $72.9 \%$ for patients in the RT group, respectively. The median RFS and OS periods were 9.21 and 25.37 months in the CT group, 7.41 and 28.85 months in the TACE group, and 25.47 and 72.54 months in the RT group, respectively. RT group showed a significantly longer RFS than the CT and TACE group (RT vs CT, $P<0.001$; RT vs TACE, $P=0.011$; Figure 1A). Likewise, patients in the RT group has longer OS compared with the CT and TACE group (RT vs CT, $P$ $<0.001$; RT vs TACE, $P=0.034$; Figure 1B). However, the RFS and OS rates between the TACE and CT groups were not significant (Figure 1A and 1B). The cumulative 1 -, 2-, 3-year RFS of all 136 patients were $46.5 \%, 32.0 \%$ and $27.0 \%$, and their cumulative OS rates were $81.1 \%$, $61.2 \%$ and $48.0 \%$, respectively.

\section{Survival analysis according to the degree of microvascular invasion and surgical margin}

The RFS and OS rates were also analyzed in the subgroups.

In the low-risk (M1) microvascular invasion with narrow surgical margin group, the 1-, 2-, 3-year RFS were $31.2,8.3$ and $0 \%$, in CT group, $61.4,51.1$ and $51.1 \%$, in TACE group, and $84.4,51.4$ and $51.4 \%$, in RT group, respectively. The 1, 2 and 3-year OS rates were 75.4 , 43.8 and $14.6 \%$ in the CT group, $87.5,75.0$ and $62.5 \%$ in the TACE group, 96.2, 90.5 and $77.6 \%$ in the RT group, respectively. RT group showed a significant longer RFS $(p<0.001)$ and OS $(p<0.001)$ than the TACE and CT groups (Figure 2A and 2B).

In the low-risk (M1) microvascular invasion with wide surgical margin group, the 1-, 2-, 3-year RFS were 75.5, 37.8 and $28.3 \%$, in CT group, 52.7, 39.6 and $39.6 \%$, in TACE group, and 80.0, 40.0 and $40.0 \%$, in RT group, respectively. The 1-, 2-, 3-year OS rates were 85.6, 77.0 and $57.8 \%$ in the CT group, 90.9, 70.7 and $56.6 \%$ in 
Table 1: The baseline characteristics and demographics of patients in the CT, TACE and RT groups

\begin{tabular}{|c|c|c|c|c|}
\hline \multirow[t]{2}{*}{ Variable } & \multicolumn{3}{|c|}{ Postoperative therapeutic management } & \multirow[t]{2}{*}{$p$ value } \\
\hline & $\mathrm{CT}(n=50)$ & TACE $(n=42)$ & RT $(n=44)$ & \\
\hline Age $($ mean $\pm \mathrm{SD})$ & $57.22 \pm 11.14$ & $51.38 \pm 10.89$ & $51.32 \pm 11.21$ & 0.014 \\
\hline Gender (Male/Female) & $5 / 45$ & $8 / 34$ & $5 / 39$ & 0.401 \\
\hline Operative time (min) & $210.84 \pm 71.88$ & $217.38 \pm 81.56$ & $238.93 \pm 76.34$ & 0.188 \\
\hline Operative procedure (Major/Minor) & $33 / 17$ & $24 / 18$ & $26 / 18$ & 0.652 \\
\hline $\begin{array}{l}\text { Operative method } \\
\text { (Anatomical/Non-anatomical) }\end{array}$ & $27 / 23$ & $21 / 21$ & $23 / 21$ & 0.929 \\
\hline Blood loss (ml) & $516.00 \pm 451.35$ & $506.67 \pm 493.24$ & $632.96 \pm 478.00$ & 0.378 \\
\hline Surgical margin $(\leq 1 \mathrm{~cm} />1 \mathrm{~cm})$ & $29 / 21$ & $22 / 20$ & $36 / 8$ & 0.010 \\
\hline Tumor size $(\mathrm{cm})$ & $5.71 \pm 2.60$ & $6.15 \pm 3.65$ & $5.63 \pm 2.73$ & 0.693 \\
\hline $\begin{array}{l}\text { Number of tumor } \\
\text { (single/multiple) }\end{array}$ & $46 / 4$ & $38 / 4$ & $41 / 3$ & 0.899 \\
\hline Differentiation (Well/Moderate/Poorly) & $2 / 30 / 18$ & $1 / 24 / 17$ & $1 / 24 / 19$ & 0.423 \\
\hline MVI classification (M1/M2) & $36 / 14$ & $24 / 18$ & $32 / 12$ & 0.216 \\
\hline Envelope invasion (Present/Absent) & $9 / 41$ & $8 / 34$ & $13 / 31$ & 0.344 \\
\hline Cirrhosis (Present/Absent) & $2 / 48$ & $4 / 38$ & $4 / 40$ & 0.336 \\
\hline Viral hepatitis (Negative/Positive) & $2 / 48$ & $0 / 42$ & $1 / 43$ & 0.543 \\
\hline $\begin{array}{l}\text { Preoperative AFP level } \\
(\leq 400 \mathrm{ng} / \mathrm{L} />400 \mathrm{ng} / \mathrm{L})\end{array}$ & $32 / 18$ & $25 / 17$ & $34 / 10$ & 0.186 \\
\hline Preoperative ALT (U/L) & $34.40 \pm 22.86$ & $34.14 \pm 24.28$ & $41.86 \pm 31.32$ & 0.293 \\
\hline Preoperative $\operatorname{TBIL}(\mu \mathrm{mol} / \mathrm{L})$ & $12.50 \pm 5.97$ & $12.55 \pm 5.23$ & $12.55 \pm 4.67$ & 0.998 \\
\hline Preoperative ALB (g/L) & $40.91 \pm 4.78$ & $42.87 \pm 5.47$ & $42.43 \pm 4.20$ & 0.121 \\
\hline Preoperative PALB (mg/dL) & $18.16 \pm 5.49$ & $19.43 \pm 5.72$ & $20.30 \pm 5.55$ & 0.178 \\
\hline Preoperative PTa (\%) & $83.97 \pm 10.70$ & $84.41 \pm 10.31$ & $80.97 \pm 9.53$ & 0.230 \\
\hline Preoperative Child-Pugh (A/B) & $50 / 0$ & $42 / 0$ & $44 / 0$ & - \\
\hline
\end{tabular}
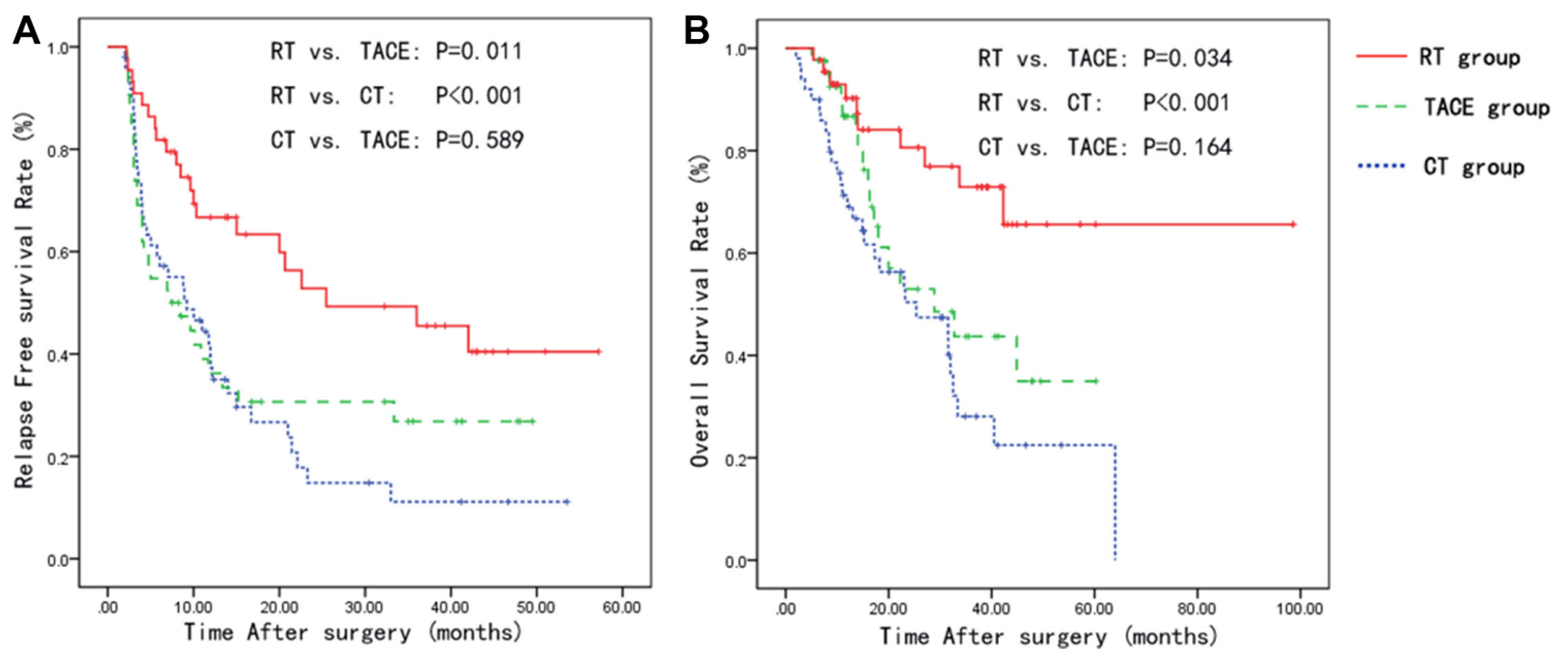

Figure 1: Kaplan-Meier curves for the relapse-free survival and overall survival in the CT, TACE and RT groups. Abbreviations: CT: Conservative therapy; TACE: Trans catheter arterial chemoembolization; RT: Radiation therapy. 
TACE group, 100.0, 80.0 and $60.0 \%$ in the RT group, respectively. There was no significant difference between the groups in the RFS and OS (Figure 2C and 2D).

In the high-risk (M2) microvascular invasion and narrow surgical margin group, the 1-, 2-, 3-year RFS rates were $0 \%, 0 \%$ and $0 \%$, in CT group; $0 \%, 0 \%$ and $0 \%$, and in TACE group; 27.8, 27.8 and $27.8 \%$, in RT group, respectively. The 1-, 2-, 3-year OS rates were 25, 25 and $0 \%$ in CT group, 60.0, 24.2 and $24.2 \%$ in the TACE group, and $70.0,52.5$ and $26.2 \%$ in the RT group, respectively. The median RFS and OS period was 3.12 and 7.77 months in CT group; 3.15 and 15.00 months in TACE group; and 9.64 and 33.74 months in RT group, respectively. RT group showed a significant better RFS $(p=0.020)$ and OS $(p=0.022)$ than the other 2 groups (Figure $3 \mathrm{~A}$ and $3 \mathrm{~B})$.

In the high-risk (M2) invasion and wide surgical margin group, the 1-, 2-, 3-year RFS rates were $0 \%, 0 \%$ and $0 \%$, in CT group; $19.0 \%, 0 \%$ and $0 \%$, and in TACE group; $0 \%, 0 \%$ and $0 \%$, in RT group, respectively. The 1-, 2-, 3-year OS rates were 66.7, 44.4 and $22.2 \%$ in CT group, $100.0,0.0$ and $0.0 \%$ in the TACE group, and 66.7 , 0.0 and $0.0 \%$ in the RT group, respectively. The median
RFS and OS duration was 3.54 and 17.28 months in the CT group; 8.39 and 16.00 months in the TACE group; and 4.72 and 8.48 months in the RT group, respectively. There was no significant difference between the groups in the RFS and OS (Figure 3C and 3D).

\section{Recurrence pattern in CT, TACE and RT groups}

Recurrence was observed in 89 of 136 patients. The incidence of marginal, non-marginal and extrahepatic recurrence was 4, 27 and 8 in CT group, 3, 19 and 7 in TACE group and 3, 13 and 5 in RT group, respectively. There was no significant difference in recurrent pattern between the three groups. The details of location of recurrent tumor were shown in Table 2.

\section{Prognostic factors affecting clinical outcomes}

The univariate Cox regression analysis indicated that blood loss, tumor size, MVI classification, envelope invasion, serum AFP level, and postoperative treatment strategies as independent risk factors that influenced
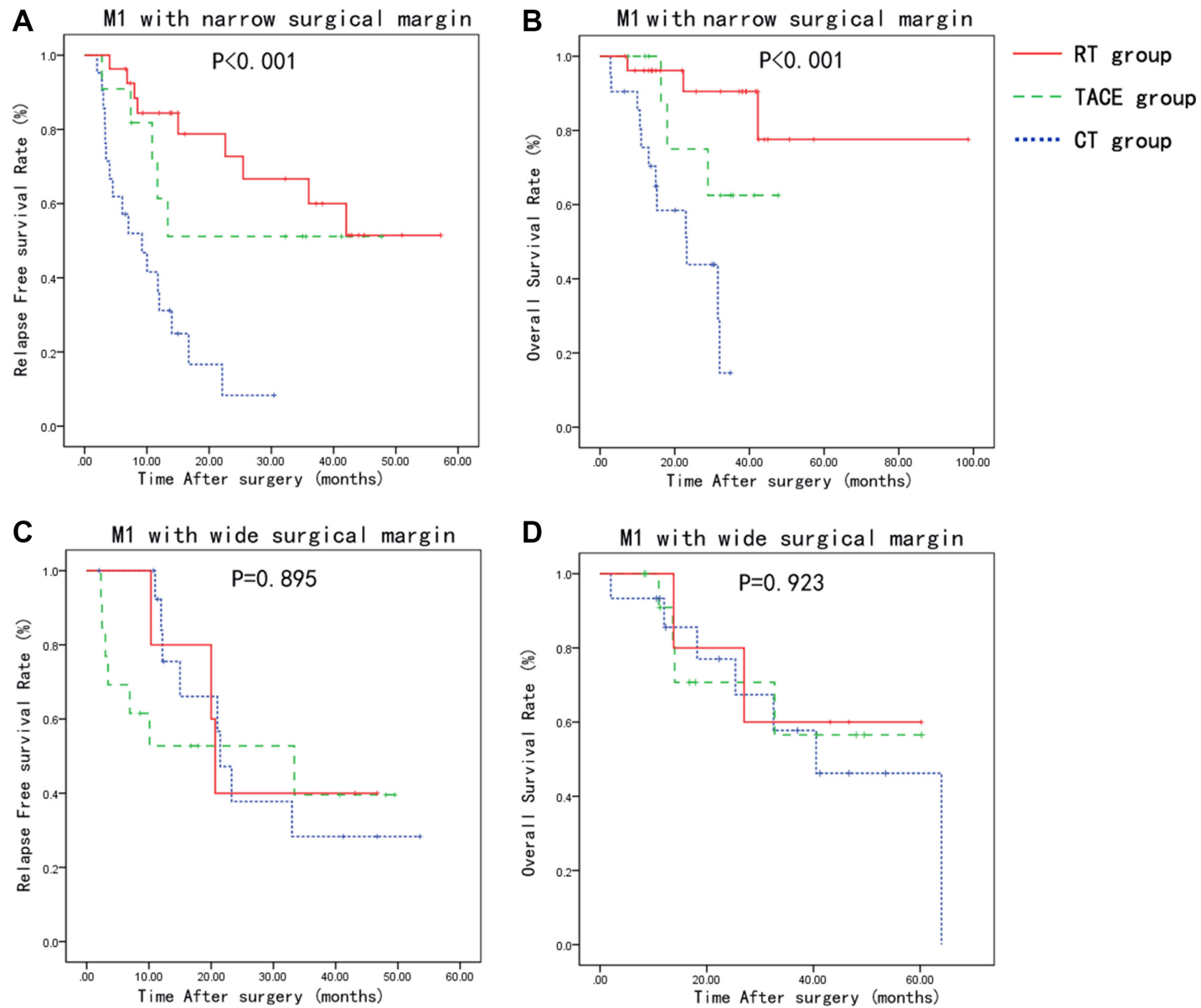

Figure 2: Relapse-free survival and overall survival curves of patients stratified based on the MVI type and surgical margin width. Patients with low-risk MVI (M1) and narrow surgical margin has improved RFS and OS with adjuvant radiotherapy compared to CT and TACE group (A, B). There was no significant difference between the groups in the RFS and OS with low-risk MVI (M1) and wide surgical margin (C, D). 
Table 2: Pattern of recurrence in the CT, TACE and RT groups

\begin{tabular}{lcccc}
\hline Pattern of recurrence & \multicolumn{2}{c}{ Postoperative therapeutic management } & \multirow{P}{*}{ value } \\
\cline { 2 - 4 } & $\mathbf{C T}(\boldsymbol{n}=\mathbf{5 0})$ & TACE $(\boldsymbol{n}=\mathbf{4 2})$ & RT $(\boldsymbol{n}=\mathbf{4 4})$ & \\
\hline Total & 39 & 29 & 21 & - \\
Intrahepatic recurrence & & & & 0.977 \\
$\quad$ Marginal & 4 & 3 & 3 & - \\
$\quad$ Non-marginal & 27 & 19 & 13 & - \\
Extrahepatic recurrence & 8 & 7 & 5 & - \\
\hline
\end{tabular}

RFS (Table 3); while tumor size, MVI classification, envelope invasion, serum AFP level, and postoperative treatment strategies were the independent factors that influenced OS (Table 4). The multivariate analysis revealed that postoperative treatment strategies, surgical margin, envelope invasion and MVI classification were the independent prognostic factors associated with RFS (Table 3); while, postoperative treatment strategies, tumor size and MVI classification were the independent prognostic factors associated with OS (Table 4).

\section{DISCUSSION}

Surgical resection is considered as the gold standard treatment modality for patients with preserved liver function and without portal hypertension. Unfortunately, the long-term survival after hepatectomy is not satisfactory because of the high incidence of intrahepatic tumor recurrence. MVI has been reported to be the most independent factor associated with recurrence and significantly affects the RFS and OS following curative resection or transplantation $[8-10,14]$. Zhao et al. reviewed 266 patients with multinodular $\mathrm{HCC}$ treated with surgical resection and found that the 3-year OS rate was $16 \%$ and $58 \%$ with and without MVI, respectively [15]. Rodriguez-Peralvarez et al. performed a meta-analysis to study the prognostic impact of MVI and found that patients with MVI had significantly reduced disease-free and OS at 3- and 5-years after liver resection and transplantation [7].

\section{Postoperative treatment strategies}

Various postoperative adjuvant therapies including TACE, radiotherapy, conservative and molecular targeted
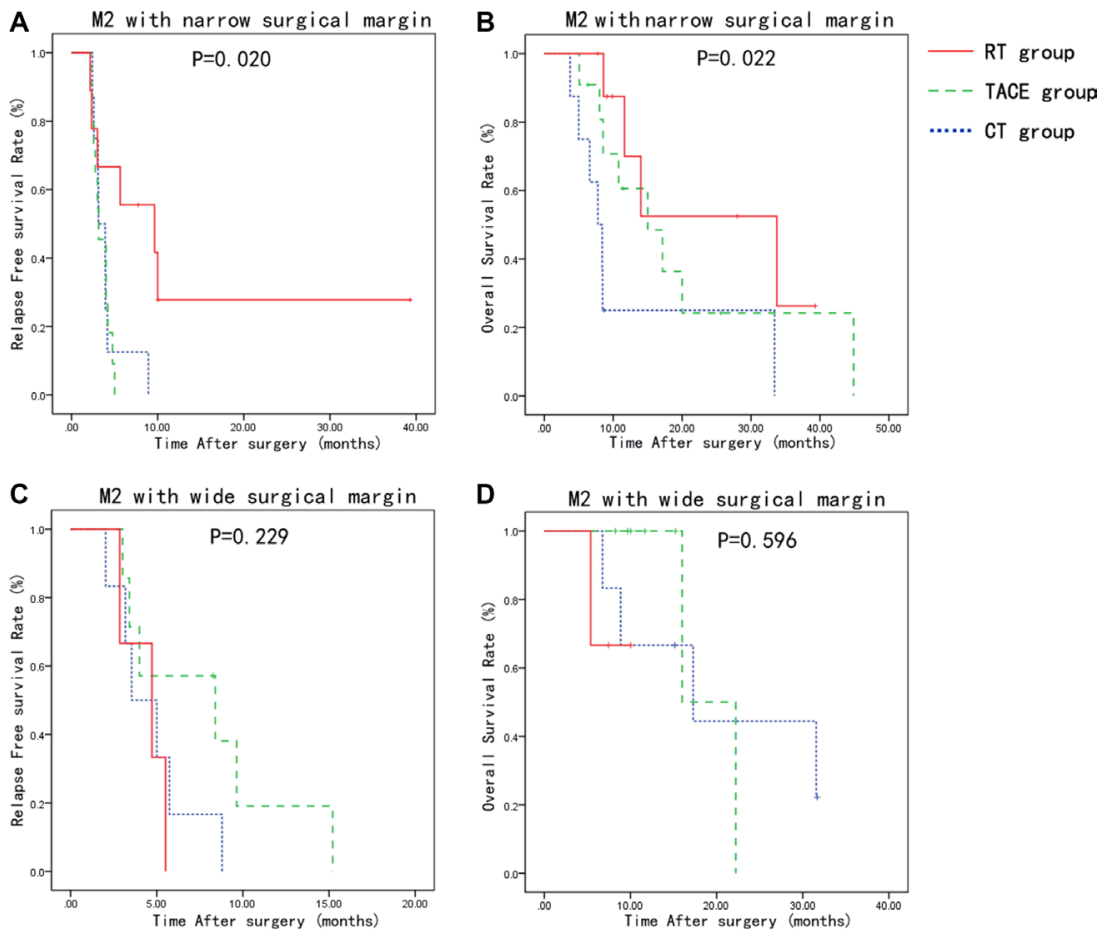

Figure 3: Relapse-free survival and overall survival curves of patients stratified by grade of MVI and surgical margin. Patients with high-risk MVI (M2) and narrow surgical margin has improved RFS and OS with adjuvant radiotherapy compared to CT and TACE group (A, B). There was no significant difference between the groups in the RFS and OS with high-risk MVI (M2) and wide surgical margin $(\mathbf{C}, \mathbf{D})$. 
Table 3: The univariate and multivariate regression analysis to identify prognostic factors for RFS

\begin{tabular}{lcccc}
\hline Variable & \multicolumn{2}{c}{ Univariate analysis } & \multicolumn{2}{c}{ Multivariate analysis } \\
\cline { 2 - 5 } & HR (95\% CI) & $\boldsymbol{p}$ value & HR (95\% CI) & $\boldsymbol{p}$ value \\
\hline RFS & $0.993(0.975-1.010)$ & 0.398 & - & - \\
Age & $1.592(0.769-3.295)$ & 0.210 & - & - \\
Gender & $1.001(0.999-1.004)$ & 0.308 & - & - \\
Operative time & $1.104(0.722-1.686)$ & 0.649 & - & - \\
Operative procedure & $1.108(0.731-1.679)$ & 0.629 & - & - \\
Operative method & $1.000(1.000-1.001)$ & 0.011 & - & - \\
Blood loss & $0.955(0.621-1.469)$ & 0.834 & $0.548(0.343-0.875)$ & 0.012 \\
Surgical margin & $1.616(1.085-1.243)$ & $<0.001$ & - & - \\
Tumor size & $1.116(0.539-2.309)$ & 0.768 & - & - \\
Number of tumors & $1.118(0.802-1.557)$ & 0.512 & - & - \\
Differentiation & $5.389(3.382-8.585)$ & $<0.001$ & $5.907(3.543-9.848)$ & $<0.001$ \\
MVI classification & $3.032(1.568-5.863)$ & 0.001 & $2.868(1.442-5.704)$ & 0.003 \\
Envelope invasion & $1.362(0.551-3.368)$ & 0.503 & - & - \\
Cirrhosis & $1.478(0.785-2.783)$ & 0.226 & - & - \\
Viral hepatitis & $1.813(1.369-2.402)$ & $<0.001$ & - & - \\
AFP level & & 0.004 & & $<0.001$ \\
Postoperative Treatment strategy & $0.865(0.534-1.402)$ & 0.557 & $0.633(0.385-1.041)$ & 0.072 \\
TACE & $0.416(0.243-0.711)$ & 0.001 & $0.278(0.154-0.501)$ & $<0.001$ \\
RT & & & &
\end{tabular}

therapies are used to decrease $\mathrm{HCC}$ recurrence and thus prolong OS. However, the outcomes of these interventions are variable and no adjuvant therapy have been universally accepted as being effective in reducing recurrence after hepatectomy [16-21]. Moreover, studies comparing these adjuvant therapies are rare and only few studies take the MVI into consideration. The development of radiation therapy techniques, such as three-dimensional conformal or IMRT has facilitated the delivery of high radiation doses to specified target area without affecting the overall liver function $[22,23]$. Wang et al. investigated the benefit of postoperative IMRT following narrow margin hepatectomy for $\mathrm{HCC}$ close to major vessels and found that IMRT improved 3-year overall and diseasefree survival without any instance of liver damage [12]. Contrarily, the effect of adjuvant radiotherapy in patients who underwent narrow margin hepatectomy for centrally located HCC found no significant difference between the groups in the overall and recurrence-free survival at 3- and 5-years [13]. In our study, adjuvant RT showed a significantly improved RFS and OS compared to TACE and $\mathrm{CT}$, which implies postoperative RT, could eliminate residual micro-metastasis-foci in the remnant liver.

The purpose of postoperative adjuvant TACE is to eliminate small intrahepatic metastases that may not have been detected preoperatively $[24,25]$. But the effects of postoperative TACE and range of applications is still a debate [26]. A retrospective study with 2436 HCC patients showed that postoperative adjuvant TACE had no effect on postponing or eliminating late recurrence [27]. A meta-analysis reported that postoperative TACE significantly improved disease free survival and OS compared to control when the mean tumor size was bigger than $5 \mathrm{~cm}$ [28]. The superiority of adjuvant TACE over hepatectomy alone was demonstrated in another meta-analysis where postoperative TACE lessened tumor recurrence for patients with multiple nodules of $>5 \mathrm{~cm}$ or macrovascular invasion [29]. Nevertheless, few studies take the MVI as an independent prognosis factor into consideration to evaluate the effect of postoperative adjuvant TACE. Although, a single center retrospective study showed that postoperative adjuvant TACE may be beneficial for HCC patients with MVI; it suggested that further randomized controlled studies are needed to make a definitive conclusion [30]. In our analysis, RFS and OS was significantly shorter in patients with postoperative adjuvant TACE compared to postoperative adjuvant RT, but no significant difference between adjuvant TACE and conservative treatment was found. These results suggested that postoperative adjuvant TACE could not be effective in eliminating residual micro-metastasis-foci in the remnant liver. The possible explanations for this result could be that MVI cannot be clearly stained during the procedure of TACE, thus patients with MVI have more possibility of undetectable micro metastasis foci and TACE can't identify and make definite elimination of 
Table 4: The univariate and multivariate regression analysis to identify prognostic factors for OS

\begin{tabular}{lcccc}
\hline Variable & \multicolumn{2}{c}{ Univariate analysis } & \multicolumn{2}{c}{ Multivariate analysis } \\
\cline { 2 - 5 } & HR (95\% CI) & $\boldsymbol{p}$ value & HR (95\% CI) & $\boldsymbol{p}$ value \\
\hline OS & $0.993(0.972-1.015)$ & 0.524 & - & - \\
Age & $1.194(0.511-2.788)$ & 0.682 & - & - \\
Gender & $1.001(0.998-1.004)$ & 0.411 & - & - \\
Operative time & $1.253(0.742-2.115)$ & 0.400 & - & - \\
Operative procedure & $0.997(0.590-1.685)$ & 0.991 & - & - \\
Operative method & $1.000(1.000-1.001)$ & 0.063 & - & - \\
Blood loss & $0.891(0.516-1.538)$ & 0.678 & - & 0.001 \\
Surgical margin & $1.178(1.087-1.275)$ & $<0.001$ & $1.152(1.056-1.256)$ & - \\
Tumor size & $0.393(0.096-1.614)$ & 0.195 & - & - \\
Number of tumors & $1.256(0.812-1.944)$ & 0.305 & - & -0.001 \\
Differentiation & $3.662(2.130-6.297)$ & $<0.001$ & $3.117(1.771-5.486)$ & - \\
MVI classification & $3.039(1.213-7.617)$ & 0.018 & - & - \\
Envelope invasion & $0.766(0.275-2.134)$ & 0.610 & - & - \\
Cirrhosis & $1.596(0.660-3.860)$ & 0.300 & - & - \\
Viral hepatitis & $1.648(1.173-2.315)$ & 0.004 & - & - \\
AFP level & & 0.002 & -0.001 \\
Postoperative Treatment strategy & $0.648(0.356-1.179)$ & 0.155 & $0.423(0.221-0.810)$ & 0.009 \\
TACE & $0.276(0.134-0.567)$ & 0.000 & $0.279(0.136-0.574)$ & 0.001 \\
RT & & & &
\end{tabular}

residual tumor. Second, targeting to the tumor bed means extending TACE to all liver segments relative to tumor, it would severely damage liver function due to its adverse effects, and the increasing complication might increase risk of recurrence. However, the conformal or intensity modulated radiotherapy, concentrates irradiation precisely to tumor bed area and selectively spares the normal liver tissues, thus limiting the adverse effects when the tumor bed with residual micro-metastasis-foci receive sufficient radiation $[12,13]$.

\section{MVI classification and surgical margin}

The feature of MVI encompasses a wide spectrum such as distance from the invaded vessel to tumor edge, the number of invaded microscopic vessels, intravascular floating tumor clusters and small vascular intratumoral spaces [31]. Each feature has different prognostic significance. Recent studies have shown that invasion of vessels $\geq 1 \mathrm{~cm}$ from the tumor capsule and the number of invaded vessels $\geq 5$ is able to stratify patients with different risks of survival [31,32]. MVI can be classified into two different categories that are inversely correlated with time to recurrence and survival [33]. In our study, uni- and multivariate analysis showed that MVI classification was independent factor either for RFS or OS. These results suggested that classification systems could distinguish risk features more accurately. However, since
MVI disseminate mainly via portal venous branches and spread along as well as against the direction of the portal venous flow, the incidence of MVI was closely related to the distance from the tumor capsule [34]. A few studies had found that persistent residual microscopic lesion in the remnant liver tissues occur commonly around the primary tumor [23, 35-37] and MVI beyond $1 \mathrm{~cm}$ from the tumor capsule is very rare [31]. Thus resection margin of at least $1.0 \mathrm{~cm}$ is preferable to eradicate microscopic lesions and theoretically gives a higher potential for the majority of patients to reduce recurrence [38, 39]. Some studies even suggested to ensure a safe resection margin $(>2 \mathrm{~cm})$ for both anatomic and non-anatomic hepatectomy $[34,40]$. On the other hand, centrally located HCC lesions that adjoin main vasculatures make the wide margin hepatectomy impossible; preserving as much nontumorous liver parenchyma as possible is also an important consideration for patients with cirrhotic liver. Thus, narrow margin resection is the only option [41-44] and the addition of effectual postoperative treatment is essential clinical required.

In our study, we conducted subgroup analysis according to MVI classification and surgical margin width. For patients of narrow surgical margin, postoperative radiotherapy yield survival outcomes significantly superior than TACE or conservative treatment regardless MVI classification. Whereas, no significant difference in the survival outcomes between the three groups of 
patients with wide surgical margin, independent of MVI classification. Based on our data, we deduced that postoperative radiotherapy might control persistent residual microscopic lesions in the remnant liver tissue in the narrow margin patients.

\section{Limitations}

The retrospective nature and the small sample size are the limitations of this study. However, the data of our study does provide rationale for developing a prospective study. Thus, our results should be validated in a randomized, controlled trial with large sample size to make a definitive conclusion on the advantages of adjuvant RT over adjuvant TACE and CT in HCC patients with MVI.

\section{MATERIALS AND METHODS}

\section{Patients}

The database of the National Cancer Center/ Cancer Hospital, Chinese Academy of Medical Sciences and Peking Union Medical College was retrospectively reviewed. A total of 646 patients who underwent hepatic resection by the same team for HCC between July 2008 and December 2015 were considered for inclusion in this study. Only patients who met all of the following eligible criteria were studied: (1) primary lesion treated with curative surgical liver resection (microscopically surgical margin free of tumor); (2) MVI were proven by postoperative pathology but without macro-vascular invasion; (3) no tumor rupture and hemorrhage before and during resection; (4) no liver failure or severe complications/adverse events after surgery within 1 month; (5) no postoperative death within 3 months; (6) preoperative liver function was Child-Pugh A degree; (7) absence of previous or simultaneous malignant tumor/ diseases; (8) patients with continuous follow-up records until death or censored time. The study was conducted in accordance with the principles of Good Clinical Practice and Declaration of Helsinki Guidelines. All the study protocols are approved by the ethics committee of National Cancer Center/Cancer Hospital, Chinese Academy of Medical Sciences and Peking Union Medical College. The requirement of informed consent was waived considering the retrospective nature of the study.

The 136 patients who met the above criteria were divided into 3 groups according to different postoperative treatments: (i) conservative treatment group (CT group, $n=50$ ), consisting of patients treated only with supportive therapy such as nutritional and anti-HBV/HCV therapy; (ii) transcatheter arterial chemoembolization (TACE group, $n=42$ ), consisting of patients treated with TACE within 2 month after surgery and supportive therapy; (iii) radiotherapy group (RT group, $n=44$ ), consisting of patients treated with three-dimensional conformal or intensity modulated radiotherapy within 2 month after surgery and supportive therapy.

\section{Postoperative evaluation}

Considering the long-standing discrepancy in definition and grading of MVI, [7] all 136 patients' histopathological sections were retrospectively collected and reviewed by professional pathologists from our center. The pathological diagnosis and classification of MVI was based on Practice guidelines for the pathological diagnosis of primary liver cancer: 2015 update [31]. The classification of MVI is defined as follows: M0: no MVI; M1 (low-risk): MVI of $<5$ and at $\leq 1 \mathrm{~cm}$ away from the adjacent liver tissues; and M2 (high-risk): MVI of $>5$ or at $>1 \mathrm{~cm}$ away from the adjacent liver tissues [31]. For the subgroup analysis, the patients were stratified according to MVI classification (M1 or M2) and surgical margin width (more or less than $1 \mathrm{~cm}$ ) into four categories: M1 with narrow surgical margin $(n=59)$, M1 with wide surgical margin $(n=33)$, M2 with narrow surgical margin $(n=28)$ and M2 with wide surgical margin $(n=16)$.

\section{Procedures of CT, TACE and RT}

Nutritional therapy and anti-HBV/HCV therapy was given to the entire patient during postoperative period as conservative treatment to improve liver function, block the process of liver cirrhosis and prevent recurrence. Adjuvant TACE was given only once as prevention measure within two months after the operation. The Seldinger's technique of arterial embolization was administered as standard TACE procedure. After suspicious residual tumor stain was identified, infusion of a mixture of $20-30 \mathrm{mg}$ of Adriamycin and 5-10 $\mathrm{mL}$ of lipiodol was performed after the arteries supplying the area of tumor were catheterized superselectively. Sufficient amount of emulsion and 2-3$\mathrm{mm}$ strips of Gelfoam were delivered to the suspicious residual tumor area until complete flow stagnation was achieved. For patients who had no definite residual tumor stain, TACE has been given to tumor bed area in prophylaxis. Three-dimensional conformal or intensity modulated radiotherapy plans were generated for RT procedure. The clinical treatment volume was defined as the tumor cutting bed expands a $1-\mathrm{cm}$ margin, and it was added by 0.5 to $1 \mathrm{~cm}$ for the final planning treatment volume. The target total dose was 54-60 Gy, which was delivered by $2 \mathrm{~Gy} /$ fraction, 5 fractions per week.

\section{Follow up}

After discharge from hospital, all patients were followed-up quarterly in the first 2 years and at 6-month intervals thereafter till the last follow-up (December 2016). Alpha-fetoprotein (AFP), liver function, chest X-ray, enhanced computed tomography and/or enhanced magnetic 
resonance imaging were performed during the follow-up visit. The HCC recurrence was diagnosed based on typical imaging findings and/or continually increased serum AFP. Biopsies were undertaken to achieve histopathology or cytopathology evidence but were not necessarily for the assessment of recurrences. The RFS and OS are the primary and secondary outcomes respectively.

RFS was defined as the time interval between the dates of surgery and the date of the first detected recurrence or censored on the date of the last follow-up. OS was recorded as time period from the date of surgery to the date of death caused by HCC recurrence or censored on the date of the last follow-up.

\section{Treatment for tumor recurrence}

The treatment strategy for recurrence of HCC was determined by multidisciplinary team mainly based on the comprehensive consideration of tumor characteristics, liver function and general condition. Local curative treatment consisted of hepatectomy and radiofrequency ablation (RFA); regional or systemic palliative treatment, such as TACE, radiation therapy, molecular targeted therapy and chemotherapy was performed as alternative methods for recurrence treatment.

\section{Statistical analysis}

Categorical variables were compared using Pearson Chi-square test or Fisher's exact test as appropriate. Continuous variable are expressed as the mean \pm standard deviation and compared using one-way analysis of variance (ANOVA). Univariate and multivariate Cox proportional hazards regression analysis of the clinicopathological parameters were performed to identify the independent prognostic factors to RFS and OS. The $P$ values were calculated using the Wald test. Survival analysis was conducted using the Kaplan-Meier methods, and comparisons were performed between groups and subgroups using a log-rank test. Statistical analysis was performed using IBM SPSS software (Version 19.0) and $P$ value $<0.05$ was considered statistically significant.

\section{CONCLUSIONS}

In conclusion, the findings demonstrate that radiotherapy following hepatectomy could result in better survival outcomes for HCC patients with MVI than postoperative TACE or conservative treatment based on survival outcomes. This treatment strategy might especially be effective for patients with narrow surgical margin.

\section{Author contributions}

L.W., W.W., X.Y., and J.W., conceptualized and designed the study. W.R., F.W., B.C., M.L., S.L., and Y.L., were involved in acquisition, analysis and interpretation of data. All the authors contributed in the preparation of initial draft, critical revision of the manuscript for important intellectual content and final approval for submission to the journal.

\section{CONFLICTS OF INTEREST}

The authors have no conflicts of interest to declare.

\section{FUNDING}

This work was supported by Beijing Hope Run Special Fund of Cancer Foundation of China (LC2015A12) and PUMC Youth Fund/Fundamental Research Funds for the Central Universities (3332016031).

\section{REFERENCES}

1. Ferlay J, Soerjomataram I, Dikshit R, Eser S, Mathers C, Rebelo M, Parkin DM, Forman D, Bray F. Cancer incidence and mortality worldwide: sources, methods and major patterns in GLOBOCAN 2012. Int J Cancer. 2015; 136:E359-386.

2. Zhu RX, Seto WK, Lai CL, Yuen MF. Epidemiology of Hepatocellular Carcinoma in the Asia-Pacific Region. Gut Liver. 2016; 10:332-9.

3. Mittal S, El-Serag HB. Epidemiology of hepatocellular carcinoma: consider the population. J Clin Gastroenterol. 2013; 47:S2-6.

4. Wang FS, Fan JG, Zhang Z, Gao B, Wang HY. The global burden of liver disease: the major impact of China. Hepatol Baltim Md. 2014; 60:2099-108.

5. Liu M, Wang L, Zhu H, Rong W, Wu F, Liang S, Xu N, $\mathrm{Wu}$ J. A Preoperative Measurement of Serum MicroRNA125b May Predict the Presence of Microvascular Invasion in Hepatocellular Carcinomas Patients. Transl Oncol. 2016; 9:167-72.

6. Shindoh J, Hasegawa K, Inoue Y, Ishizawa T, Nagata R, Aoki T, Sakamoto Y, Sugawara Y, Makuuchi M, Kokudo N. Risk factors of post-operative recurrence and adequate surgical approach to improve long-term outcomes of hepatocellular carcinoma. HPB. 2013; 15:31-9.

7. Rodríguez-Perálvarez M, Luong TV, Andreana L, Meyer T, Dhillon AP, Burroughs AK. A systematic review of microvascular invasion in hepatocellular carcinoma: diagnostic and prognostic variability. Ann Surg Oncol. 2013; 20:325-39.

8. Barreto SG, Brooke-Smith M, Dolan P, Wilson TG, Padbury RTA, Chen JWC. Cirrhosis and microvascular invasion predict outcomes in hepatocellular carcinoma. ANZ J Surg. 2013; 83:331-5.

9. Moon JI, Kwon CH, Joh JW, Choi GS, Jung GO, Kim JM, Shin M, Choi SJ, Kim SJ, Lee SK. Primary versus 
salvage living donor liver transplantation for patients with hepatocellular carcinoma: impact of microvascular invasion on survival. Transplant Proc. 2012; 44:487-93.

10. Fan LF, Zhao WC, Yang N, Yang GS. Alpha-fetoprotein: the predictor of microvascular invasion in solitary small hepatocellular carcinoma and criterion for anatomic or nonanatomic hepatic resection. Hepatogastroenterology. 2013; 60:825-36.

11. Du M, Chen L, Zhao J, Tian F, Zeng H, Tan Y, Sun H, Zhou $\mathrm{J}$, Ji Y. Microvascular invasion (MVI) is a poorer prognostic predictor for small hepatocellular carcinoma. BMC Cancer. $2014 ; 14: 38$.

12. Wang WH, Wang Z, Wu JX, Zhang T, Rong WQ, Wang LM, Jin J, Wang SL, Song YW, Liu YP, Ren H, Fang H, Wang WQ, et al. Survival benefit with IMRT following narrowmargin hepatectomy in patients with hepatocellular carcinoma close to major vessels. Liver Int. 2015; 35:2603-10.

13. Yu W, Wang W, Rong W, Wang L, Xu Q, Wu F, Liu L, Wu J. Adjuvant radiotherapy in centrally located hepatocellular carcinomas after hepatectomy with narrow margin $(<1 \mathrm{~cm})$ : a prospective randomized study. J Am Coll Surg. 2014; 218:381-92.

14. McHugh PP, Gilbert J, Vera S, Koch A, Ranjan D, Gedaly R. Alpha-fetoprotein and tumour size are associated with microvascular invasion in explanted livers of patients undergoing transplantation with hepatocellular carcinoma. HPB. 2010; 12:56-61.

15. Zhao WC, Fan LF, Yang N, Zhang HB, Chen BD, Yang GS. Preoperative predictors of microvascular invasion in multinodular hepatocellular carcinoma. Eur J Surg Oncol. 2013; 39:858-64.

16. Jeng WJ, Lin CC, Chen WT, Sheen IS, Lin CY, Lin SM. Adjuvant therapy for hepatocellular carcinoma after curative treatment. Dig Dis. 2014; 32:747-54.

17. Kobayashi $T$, Ishiyama $K$, Ohdan $H$. Prevention of recurrence after curative treatment for hepatocellular carcinoma. Surg Today. 2013; 43:1347-54.

18. Lei J, Zhong J, Hao J, Liu Z, Zhang P, Wu L, Yan L, Zhu J, Zeng Y, Li B, Wen T, Wang W. Hepatocellular carcinoma cases with high levels of c-Raf-1 expression may benefit from postoperative adjuvant sorafenib after hepatic resection even with high risk of recurrence. Oncotarget. 2016; 7:42598-607. https://doi.org/10.18632/oncotarget.3799.

19. Peng BG, He Q, Li JP, Zhou F. Adjuvant transcatheter arterial chemoembolization improves efficacy of hepatectomy for patients with hepatocellular carcinoma and portal vein tumor thrombus. Am J Surg. 2009; 198:313-8.

20. Zhong C, Guo R, Li J, Shi M, Wei W, Chen M, Zhang Y. A randomized controlled trial of hepatectomy with adjuvant transcatheter arterial chemoembolization versus hepatectomy alone for Stage III A hepatocellular carcinoma. J Cancer Res Clin Oncol. 2009; 135:1437-45.

21. Zhong JH, Du XK, Xiang BD, Li LQ. Adjuvant sorafenib in hepatocellular carcinoma: a cautionary comment of storm trial. World J Hepatol. 2016; 8:957-60.
22. Kim JY, Chung SM, Choi BO, Kay CS. Hepatocellular carcinoma with portal vein tumor thrombosis: Improved treatment outcomes with external beam radiation therapy. Hepatol Res. 2011; 41:813-24.

23. Wang W, Feng X, Zhang T, Jin J, Wang S, Liu Y, Song Y, Liu X, Yu Z, Li Y. Prospective evaluation of microscopic extension using whole-mount preparation in patients with hepatocellular carcinoma: Definition of clinical target volume for radiotherapy. Radiat Oncol. 2010; 5:73.

24. Ren ZG, Lin ZY, Xia JL, Ye SL, Ma ZC, Ye QH, Qin LX, Wu ZQ, Fan J, Tang ZY. Postoperative adjuvant arterial chemoembolization improves survival of hepatocellular carcinoma patients with risk factors for residual tumor: a retrospective control study. World J Gastroenterol. 2004; 10:2791-4.

25. Sun HC, Tang ZY. Preventive treatments for recurrence after curative resection of hepatocellular carcinoma--a literature review of randomized control trials. World J Gastroenterol. 2003; 9:635-40.

26. Li KW, Li X, Wen TF, Lu WS. The effect of postoperative TACE on prognosis of HCC: an update. Hepatogastroenterology. 2013; 60:248-51.

27. Chen X, Zhang B, Qiu S, Fan J, Ren Z, Xia J, Wang Y, Gan Y, Yin X, Ye S. [Effect of postoperative adjuvant transarterial chemoembolization on late recurrence of hepatocellular carcinoma after radical resection]. [Article in Chinese]. Zhonghua Gan Zang Bing Za Zhi. 2010; 18:599-603.

28. Cheng X, Sun P, Hu QG, Song ZF, Xiong J, Zheng QC. Transarterial (chemo)embolization for curative resection of hepatocellular carcinoma: a systematic review and metaanalyses. J Cancer Res Clin Oncol. 2014; 140:1159-70.

29. Zhong JH, Li LQ. Postoperative adjuvant transarterial chemoembolization for participants with hepatocellular carcinoma: A meta-analysis. Hepatol Res. 2010; 40:943-53.

30. Sun JJ, Wang K, Zhang CZ, Guo WX, Shi J, Cong WM, Wu MC, Lau WY, Cheng SQ. Postoperative Adjuvant Transcatheter Arterial Chemoembolization After R0 Hepatectomy Improves Outcomes of Patients Who have Hepatocellular Carcinoma with Microvascular Invasion. Ann Surg Oncol. 2016; 23:1344-51.

31. Roayaie S, Blume IN, Thung SN, Guido M, Fiel MI, Hiotis S, Labow DM, Llovet JM, Schwartz ME. A system of classifying microvascular invasion to predict outcome after resection in patients with hepatocellular carcinoma. Gastroenterology. 2009; 137:850-5.

32. Sumie S, Nakashima O, Okuda K, Kuromatsu R, Kawaguchi A, Nakano M, Satani M, Yamada S, Okamura S, Hori M, Kakuma T, Torimura T, Sata M. The Significance of Classifying Microvascular Invasion in Patients with Hepatocellular Carcinoma. Ann Surg Oncol. 2014; 21:1002-9.

33. Cong WM, Bu H, Chen J, Dong H, Zhu YY, Feng LH, Chen J, Committee G. Practice guidelines for the pathological diagnosis of primary liver cancer: 2015 update. World J Gastroenterol. 2016; 22:9279-87. 
34. Shi M, Guo RP, Lin XJ, Zhang YQ, Chen MS, Zhang CQ, Lau WY, Li JQ. Partial hepatectomy with wide versus narrow resection margin for solitary hepatocellular carcinoma: a prospective randomized trial. Ann Surg. 2007; 245:36-43.

35. Okusaka T, Okada S, Ueno H, Ikeda M, Shimada K, Yamamoto J, Kosuge T, Yamasaki S, Fukushima N, Sakamoto M. Satellite lesions in patients with small hepatocellular carcinoma with reference to clinicopathologic features. Cancer. 2002; 95:1931-7.

36. Shi $M$, Zhang CQ, Zhang YQ, Liang XM, Li JQ. Micrometastases of solitary hepatocellular carcinoma and appropriate resection margin. World J Surg. 2004; 28:376-81.

37. Zhou XP, Quan ZW, Cong WM, Yang N, Zhang HB, Zhang $\mathrm{SH}$, Yang GS. Micrometastasis in surrounding liver and the minimal length of resection margin of primary liver cancer. World J Gastroenterol. 2007; 13:4498-503.

38. Hirokawa F, Hayashi M, Miyamoto Y, Asakuma M, Shimizu T, Komeda K, Inoue Y, Uchiyama K. Outcomes and predictors of microvascular invasion of solitary hepatocellular carcinoma. Hepatol Res. 2014; 44:846-53.

39. Hu RH, Lee PH, Chang YC, Ho MC, Yu SC. Treatment of centrally located hepatocellular carcinoma with central hepatectomy. Surgery. 2003; 133:251-6.
40. Shimada K, Sakamoto Y, Esaki M, Kosuge T. Role of the width of the surgical margin in a hepatectomy for small hepatocellular carcinomas eligible for percutaneous local ablative therapy. Am J Surg. 2008; 195:775-81.

41. Chang CM, Wei CK, Lee CH, Tseng KC, Lin CW, Yin WY. Long-term follow-up of central hepatocellular carcinoma in comparison with non-central hepatocellular carcinoma. Hepatogastroenterology. 2012; 59:492-5.

42. Matsui Y, Terakawa N, Satoi S, Kaibori M, Kitade H, Takai S, Kwon AH, Kamiyama Y. Postoperative outcomes in patients with hepatocellular carcinomas resected with exposure of the tumor surface: clinical role of the no-margin resection. Arch Surg. 1960. 2007; 142:596-602.

43. Miao XY, Hu JX, Dai WD, Zhong DW, Xiong SZ. Null-margin mesohepatectomy for centrally located hepatocellular carcinoma in cirrhotic patients. Hepatogastroenterology. 2011; 58:575-82.

44. Wu J, Wang L, Liu L, Zhong Y, Rong W, Wu F, Xu Q, Wang Y, Miao C, Yu W. [Application of selective and timely regional hepatic vascular occlusion for resection of large centrally located liver tumors: report of 133 cases]. [Article in Chinese]. Zhonghua Zhong Liu Za Zhi. 2012; 34:850-4. 